\title{
ON THE SHAPE OF THE PULSAR EMISSION DIAGRAM
}

\author{
I. M. YUSIFOV \\ Institute of Physics, Azerbaijan Academy of Sciences
}

\begin{abstract}
On the basis of pulse profiles in a limited interval of wavelength for $\sim 260$ pulsars, the dependence of the shape and width of the pulsar radiation pattern on pulsar period is investigated. The relative pulse duration is compared with the ratio of numbers of single and multicomponent pulsars. It is shown that the simple circular hollow cone model does not agree with observational data. Preliminary estimates show that the young pulsars (with short periods) have an elliptical pattern which at long periods becomes circular.
\end{abstract}

\section{Introduction}

At present there is much observational data on the widths of pulses, polarization and on the other parameters of pulsars. This allows us to carry out a self-consistent investigation of the evolution of the main physical parameters of pulsars. The investigations are carried out by the Monte-Carlo simulation method. For the generation of various models it is very important to know the shape and width of the pulsar radiation beam. It is this problem that will mainly be considered in this paper. Various models and their agreement with the observational data will be considered later.

The problem is that using two observed parameters [ $W_{10}$ and $\left.(d \psi / d \phi)_{\max }\right]$, one estimates the values of three angles: $\alpha$, the angle between the magnetic and rotation axes; $\beta$, the inclination of the rotation axis with respect to the line of sight; and the conal angle of the pattern $2 \theta$. To solve this problem we introduce here a new parameter, $\delta \theta$, the thickness of the walls of hollow cone radiation beam of pulsars. Approximately the same problem has been considered by Izvekova et al. (1977). At that time, however, the analysis suffered both because the statistics were poor (only $\sim 15$ objects) and spherical effects had not been taken into account.

\section{The circular hollow cone model}

Let us assume that the pulsars have a hollow cone beam with an opening angle of $20^{\circ}$ and a thickness $\delta \theta$ as in Backer's (1976) and Rankin's (1983a) classification (figure 1). When the line of sight intersects the edge of the diagram the observer can see one pulse, but in the case $|\alpha-\beta|<\theta$, the observer can see (more or less resolved) double or multicomponent pulses depending on the value $|\alpha-\beta|$. Calculating the ratio of solid angles which are drawn in the space by the peripheral and central parts of the diagram, one can estimate the ratio of the number of pulsars with single pulses to the number of two- (and multi-) component pulsars. For angles $\alpha+\theta+\delta \theta / 2 \leq 90^{\circ}$, this ratio is

$$
K=\frac{N_{1}}{N_{2}}=\frac{\sin (\theta+\delta \theta / 2)}{\sin \theta}-1
$$

When the line of sight intersects such a diagram we observe a two-component pulse with the distance between the components $2 \phi$ and with a subpulse width $\delta \phi$ at the ten percent level (figure 1). From the observed width of two-component pulses one can derive the following ratio

$$
F(\phi)=\frac{\sin ^{2}\left(\phi_{2} / 2\right)-\sin ^{2}\left(\phi_{1} / 2\right)}{\sin ^{2}(\phi / 2)} \simeq 2 \frac{\sin (\delta \phi / 2)}{\tan (\phi / 2)}
$$

The meanings of $\phi_{1}$ and $\phi_{2}$ are seen from figure 1. Using eq.(10.23) from Manchester and Taylor's (1977) monograph, one can estimate the expected value of the ratio [eq.(2)] for a given $\alpha$ and $\beta$. After simple transformations for the expected value $F(\phi)$, we obtain following expression

$$
F(\phi)=\frac{\sin ^{2}\left(\phi_{2} / 2\right)-\sin ^{2}\left(\phi_{1} / 2\right)}{\sin ^{2}(\phi / 2)}=\frac{2 \sin \theta \sin (\delta \theta / 2)}{\cos (\alpha-\beta)} \frac{-\cos \theta}{3(3)}
$$

By comparing the expected values of $K$ and $F(\phi)$ with the observed values in various period intervals, one can estimate $\theta$ and $\delta \theta$ and then, using the polarization data, calculate $\alpha$ and $\beta$. In order to check the possible evolution of $\theta$ and $\alpha$ we considered pulsars in six period intervals, the latter being chosen so that there were adequate and approximately equal statistics in each period interval. The results are given in table 1.

The first line shows the period intervals. The second line gives the number of pulsars in the inter$\mathrm{val}$, and the third the ratio of the number of pulsars with single pulses to the number of pulsars with multiple components. The fourth line then gives 


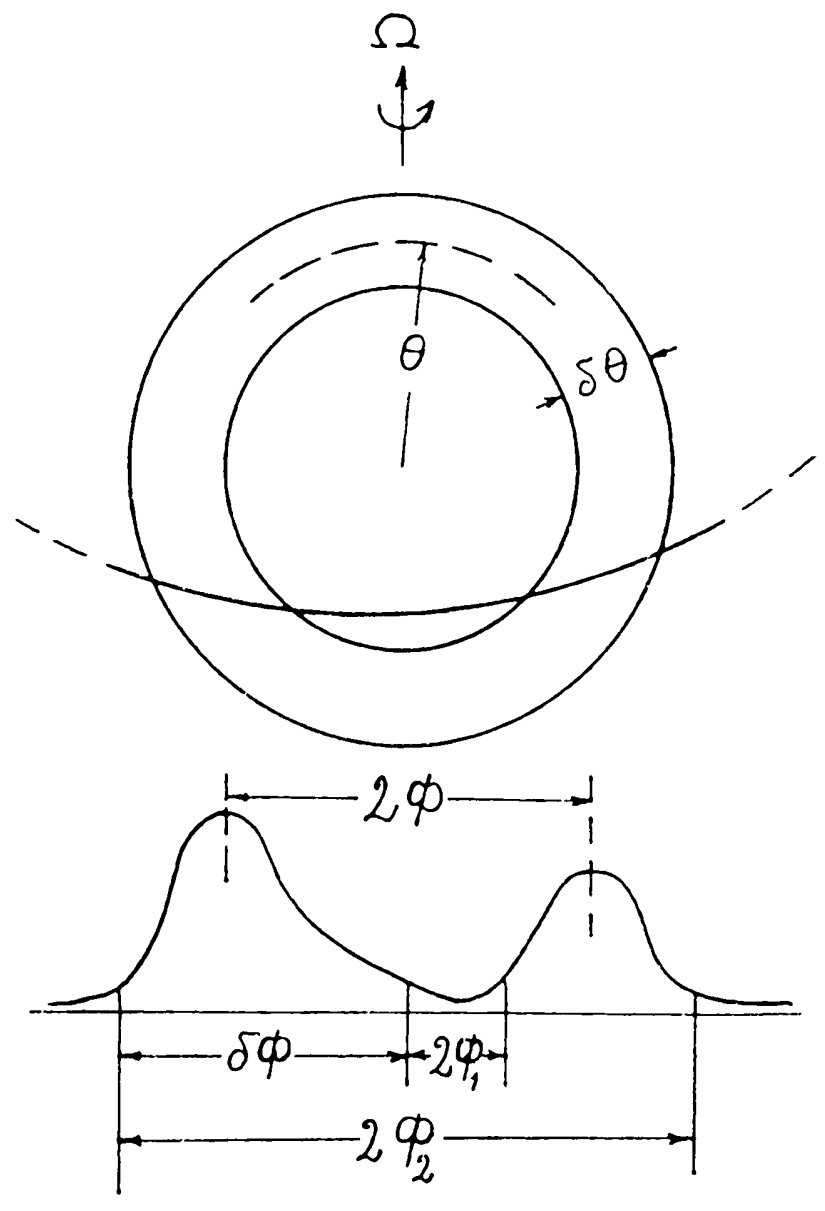

Figure 1 Schematic section of the hollow cone and twocomponent pulse visible at its intersection..

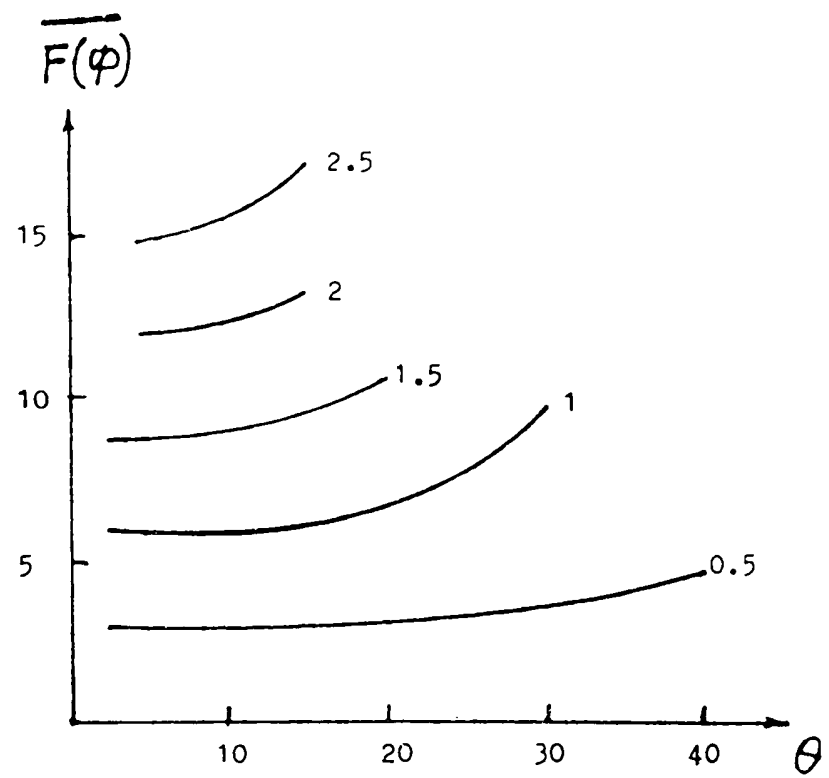

Figure 2 The dependence of the expected mean value $F(f)$ on $q$. The values of $K$ are given near the lines.

Table 1

\begin{tabular}{|c|cccccc|}
\hline$P(\mathrm{~s})$ & $0.01-0.2$ & $0.2-0.4$ & $0.4-0.6$ & $0.6-1$ & $1-2$ & $2-4$ \\
\hline$\Delta N$ & 19 & 46 & 44 & 60 & 55 & 11 \\
$K$ & 2.86 & 1.33 & 1.82 & 2 & 0.59 & 0.57 \\
$\frac{F_{0}(\phi)}{2}$ & 2.5 & 6 & 4 & 4.5 & 4 & 2.7 \\
\hline
\end{tabular}

the mean value $\overline{F_{0}(\phi)}$ in every period interval obtained from the observationally determined quantity $F(\phi)$. A diagram showing the dependence of the expected mean values $\overline{F(\phi)}$ on $\theta$ for the various values of $K$ is labelled figure 2 .

From figure 2 it is seen that the observed mean value $\overline{F_{0}(\phi)}$ is significantly less than the expected $\overline{F(\phi)}$ for a given $K$. This means that the circular hollow cone model does not agree with the observational data. If one assumes that in the vicinity of the radiation beam, active points are distributed randomly, as in Lyne and Manchester's (1988) model, visible contradictions may disappear. But in this case we must accept that in short period pulsars, single active points predominate (see table 1). Unfortunately, this model also has some difficulties. The alternative may be an elliptical diagram, which is elongated along the pulsar meridian. From the table and figure 2, it is seen that the circular cone model provides a solution only at large rotational periods. This result agrees with the conclusion of Narayan and Vivekanand (1982) and Guseinov and Yusifov (1985) that the elongated diagram at short periods transforms into a circular one for long periods. Approximate estimates show that the axial ratio of the elliptical pattern may be as high as three.

\section{Elliptical diagram}

In order to take into account the elongation of the emission pattern along the meridian, we can derive the exact dependence of the visible width of the beam $\phi$ from $\alpha, \beta, \theta$ and $\theta_{1}$ (where $\theta$ is the latitudinal width, and $\theta_{1}$ the longitudinal width of the beam). Preliminary mention of this problem was made in Guseinov and Yusifov (1985), and a more detailed derivation of this relation is given in $\mathrm{Gu}$ seinov and Yusifor (1990). Here we give only the final form of the dependence: 


$$
\begin{aligned}
\cos \phi= & \frac{\cos \theta \sqrt{\left(\cos ^{2} \beta+m^{2} \sin ^{2} \beta\right)\left(\sin ^{2} \theta+m^{2} \cos ^{2} \theta\right)-m^{2} \cos ^{2} \alpha}}{\left(\sin ^{2} \theta+m^{2} \cos ^{2} \theta-\cos ^{2} \alpha\right) \sin \beta} \\
& -\frac{\sin \alpha \cos \alpha \cos \beta}{\left(\sin ^{2} \theta+m^{2} \cos ^{2} \theta-\cos ^{2} \alpha\right) \sin \beta}
\end{aligned}
$$

where $m=\tan \theta / \tan \theta_{1}$. This is a more general formula. When $m=1$, it transforms into the formula for the circular diagram.

The results of the more detailed analysis, carried out using the Monte-Carlo method and taking into account all available data, will be published as soon as possible (Guseinov and Yusifov 1991). We would like to note here only that the comparison of theoretical models with the observations was carried out in the parameter spaces $W_{10} v s . P,(d \psi / d \phi) v s$. $P$, and $P$ vs. $\dot{P}$.

The results of calculations show that pulsars are mainly formed with short periods $(P<0.1 \mathrm{~s})$. The combined decay time of pulsars for the alignment of the axes and decay of the magnetic field is 5$10 \times 10^{6} \mathrm{yr}$.

\section{Conclusion}

We have considered $\sim 260$ pulsars with well determined integrated profiles in a limited wavelength interval. Our analysis shows that the radiation beam of short period pulsars is elongated along the meridian, and the elliptical beam axial ratio may be as high as three. But in long period pulsars the radiation beam is circular. Exclusion of pulsars with "partial cone" and "core" type profiles (which comprise $\sim 30 \%$ of total number) on the basis of polarization data does not change the main result of this analysis. Pulsars are generally born with short periods, and their decay time is $5-10 \times 10^{6} \mathrm{yr}$.

Acknowledgment: The author is grateful to Prof. O. H. Guseinov for his valuable comments and discussions. 